Int. J. Dev. Biol. 50: 393-398 (2006)

doi: $10.1387 / \mathrm{ijdb} .052030 \mathrm{vl}$

Original Article

\title{
Egg development in parthenogenetic nematodes: variations in meiosis and axis formation
}

\author{
VERA LAHL, BERND SADLER and EINHARD SCHIERENBERG* \\ Zoologisches Institut, University of Köln, Germany
}

\begin{abstract}
In the well studied model nematode Caenorhabditis elegans entrance of the sperm induces an anterior-posterior polarity in the egg and determines the orientation of the primary embryonic axis. Subsequently, fusion of two haploid gamete nuclei results in a diploid zygote as a prerequisite for normal embryogenesis. Here we analyze the establishment of embryonic polarity and diploidy in the absence of sperm in three parthenogenetic nematode species from three different families, Diploscapter coronatus (Diploscapteridae), Acrobeloides nanus (Cephalobidae) and Plectus sp. (Plectidae). We find that they not only differ from $C$. elegans in these two aspects but also from each other, indicating variant solutions for the same developmental challenges and supporting the view that the parthenogenetic mode of reproduction has been acquired multiple times independently.
\end{abstract}

KEY WORDS: nematode, embryo, parthenogenesis, meiosis, polarity

\section{Introduction}

Most higher organisms follow a gonochoristic (male/female) mode of reproduction, which is thought to give at least longterm advantages because of the continuous recombination of alleles, resulting for instance in the loss of lethal mutations (Maynard-Smith, 1978) and a better resistance to parasites (Hamilton et al., 1990). However, the advantages of sex are counterbalanced by at least short-term advantages of parthenogenetic species where each individual can reproduce and where the costs of mate search, courtship, intraspecific competition, etc. can be spared.

It is generally agreed that the gonochoristic mode is original and other variants like hermaphroditism or parthenogenesis are derived forms. Parthenogenetic reproduction has been found in various animal phyla (Mittwoch, 1978), not only in low invertebrates like rotifers and plathyhelminths or in insects but also in some vertebrate species (Cole and Townsend, 1990). Recently, complete parthenogenetic development to term has been experimentally induced in mice after overcoming "parental imprinting," which in mammals normally requires the presence of maternal and paternal genomes (Kono et al., 2004).

Parthenogenesis is frequently found in certain free-living nematode taxa. Several such species are being cultured and studied in our laboratory (Skiba and Schierenberg, 1992; Lahl et al., 2003), offering the opportunity to analyze in detail developmental peculiarities that accompany this type of repro- duction. Certain parasitic nematodes have been described in which reproduction alternates between a generation with a bisexual and one with a unisexual (parthenogenetic) mode (Viney, 1999). While it is difficult to investigate the underlying mechanism in such species they demonstrate at least that both reproductive variants can be encoded by the same genome. In addition, they suggest that a switch from one mode to the other requires only a limited number of changes in the developmental program.

During oogenesis in the internally self-fertilizing hermaphrodite $C$. elegans, oocytes arrest during meiosis and need to be induced by a sperm-derived signal to resume their meiotic program (Miller, 2001; Hajnal and Berset, 2002) in order to become haploid and be ready for fertilization. Egg cells lose their centrioles and meiotic divisions take place without them (Albertson and Thomson, 1993). The sperm then delivers the centriole necessary to generate embryonic cleavage spindles. In $C$. elegans, it is also the sperm that induces formation of the primary embryonic axis, i.e. the area of its entrance into the egg defines the posterior pole (Goldstein and Hird, 1996).

Taking $C$. elegans as a reference, it is obvious that development of parthenogenetic nematodes must require certain modifications during oogenesis and/or early embryogenesis. These include:(i) preservation or restoration of diploidy without paternal contribution, either through absent or incomplete meiosis or via compensating postmeiotic processes; (ii) establishment of egg polarity without fertilization, i.e. either by random chance

\footnotetext{
*Address correspondence to: Dr. Einhard Schierenberg. Zoological Institute, Universität Köln, Kerpener Str. 15, D-50923 Köln, Germany. Fax: +49-221-470-4987. e-mail: e.schierenberg@uni-koeln.de
} 
processes or via polarizing cues acting in the mother; (iii) formation of cleavage spindles despite the absence of a spermderived centriole requiring either survival of the original centriole, a de novo synthesis in the egg cell, or formation of centrosomes without centrioles.

The aim of this study is to gain initial insights into how developmental problems going along with the absence of sperm are overcome in parthenogenetic nematodes and whether the solutions for these problems are identical or different among the studied representatives.

\section{Results}

\section{Parthenogenesis: no sperm and only one pronucleus}

Mature adult hermaphrodites of $C$. elegans, like many other nematode species, are characterized by the presence of sperm localized in the spermatheca between the oviduct and uterus (Fig. 2A). After fertilization two haploid pronuclei form (Fig. 2B), which fuse to generate a diploid zygote. For the present study we obtained evidence that Acrobeloides nanus, Diploscapter coronatus and Plectus sp. reproduce parthenogenetically. First, we found no males in these strains, even if kept at increased temperature $\left(30^{\circ} \mathrm{C}\right)$, to favor chromosome loss (Sulston and Hodgkin, 1988). Second, we microscopically investigated adult gonads and eggs in our cultures for the presence of sperm and the number of pronuclei. In all cases, no sperm (Fig. 2C) and only one pronucleus (Fig. 2D) were found $(n=5-10 /$ strain $)$.

\section{Meiosis and formation of polar bodies}

In $C$. elegans two consecutive meiotic divisions result in the extrusion of two polar bodies (PBs) at the anterior pole, with a

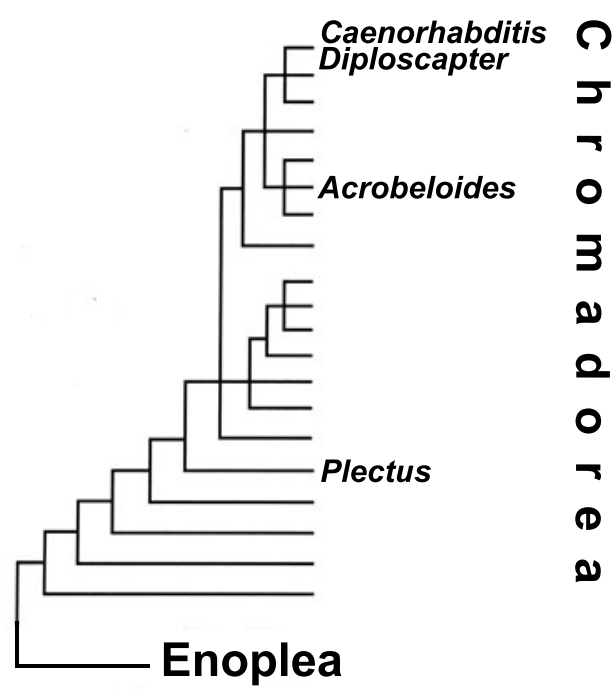

Fig. 1. Phylogenetic tree of nematodes derived primarily from SSU rDNA sequence data (after De Ley and Blaxter, 2002). According to the taxonomy of these authors, the four studied species are members of the class Chromadorea (which includes the taxon formerly classified as class Secernentea, as well as several orders formerly placed in class Adenophorea). The branching patterns within the class Enoplea (which includes the remainder of orders formerly placed in Adenophorea) is not shown here.
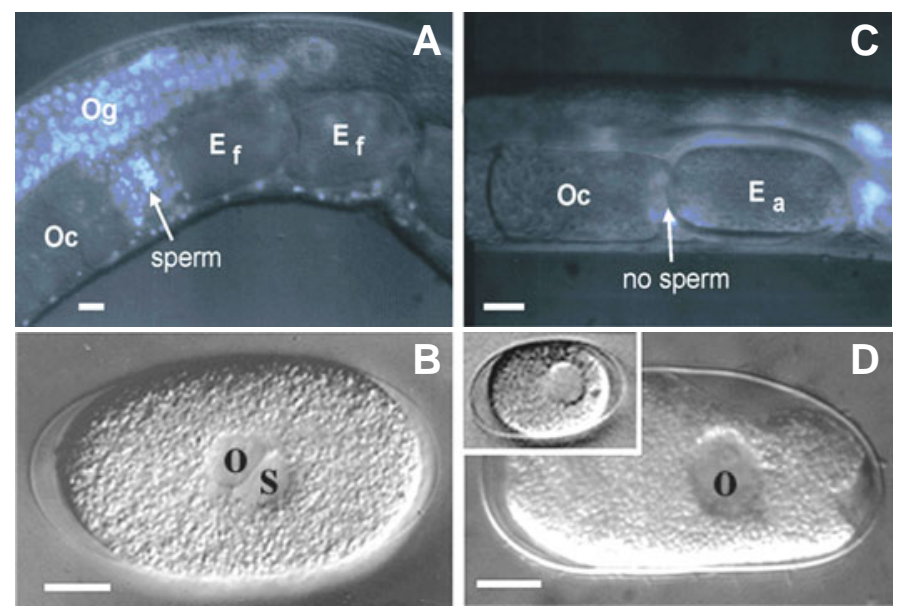

Fig. 2. Fertilization and parthenogenesis. (A) Gonad of C. elegans; small oogonia (Og) grow to form large box-shaped oocytes (Oc) which are fertilized by sperm from the same hermaphroditic mother; fertilized eggs (Ef) start cleavage in the uterus. (B) In C. elegans, oocyte- (o) and spermderived (s) pronuclei fuse to form a zygote. (C) In the parthenogenetic D. coronatus, no sperm is present, oocytes (oc) start embryogenesis after activation (Ea). (D) In 1-cell stages of the parthenogenetic A. nanus and Plectus sp. (ES 601)(insert), only a single large oocyte nucleus (o) is present. Bars, $10 \mu \mathrm{m} ;(A, C)$, combination of Nomarski optics and epifluorescence to visualize DAPI-stained nuclei; $(B, D)$ Nomarski optics.

DNA content of $2 \mathrm{c}$ and $1 \mathrm{c}$, respectively, and a haploid oocyte nucleus (Nigon et al., 1960). Looking at fluorescently labeled DNA, the first PB appears larger than the second (Fig. 3A). Thus, numbers of $\mathrm{PBs}$ and relative sizes as seen under the fluorescence microscope give an indication of the meiotic processes that occurred before.

We compared the formation of PBs in the three parthenogenetic species $A$. nanus, $D$. coronatus, and Plectus sp., and in addition in two other Acrobeloides species, one with a dioecious (SB 374) and the other with a hermaphroditic (PS1146) mode of reproduction, respectively, to the pattern found in $C$. elegans and observed noticeable differences.

In $D$. coronatus two PBs are generated, suggesting execution of a complete meiosis. However, a closer inspection of DAPIstained specimens revealed that this is not the case. In all 1-cell and many 2-cell stages only one PB is present, always localized in the area where the first cleavage furrow forms (Fig. 3B). In a minority of 2-cell stages (Fig. 3B; insert) and in most 4-cell stages two small, equal-sized PBs, positioned side-by-side, are found $(n=55)$. Analysis of living embryos $(n=4)$ confirmed that this is the result of a division of the first PB.

In $A$. nanus usually only one large PB located at the side of the egg (Fig. 3C), is formed (94/98). This indicates that only one meiotic division takes place in this species. In rare cases $(4 / 98)$ two small PBs in adjacent position were found (similar to Fig. $3 \mathrm{~B}$, insert), indicating division of the first PB. We made video recordings of living embryos to study in more detail the process of meiosis and polar body formation in $A$. nanus. In the majority of cases $(6 / 9)$ this process could not be clearly resolved as the oocyte nucleus divided into the depth of focus. However, in 3 out of the 9 cases the execution of a second meiotic division, resulting in two separate nuclei, could be observed. In these 
instances oocyte nucleus and second PB were found to fuse again.

We conclude, that the truncated meiosis described above for $A$. nanus and $D$. coronatus enables the egg cell nucleus to retain or regain a diploid status without the requirement for zygote formation (i.e. without fusion with a sperm).

In both Acrobeloides species with sperm (SB 374, $\mathrm{n}=28$; $\mathrm{PS} 1146, \mathrm{n}=32$ ) the egg cell nucleus always passes through two consecutive meiotic divisions, resulting in the generation of two PBs. During early cleavage one of them divides again (Fig. 3D). As a consequence of fertilization, two pronuclei are generated, like in $C$. elegans (Fig. 2B), which fuse to form a zygote.

Also in Plectus sp. (ES 601), two PBs are formed (Fig. 3E; $\mathrm{n}=68$ ) as meiotic by-products, despite its parthenogenetic mode of reproduction. Only the first meiotic division is executed inside the mother but after eggs have been laid, meiosis is completed with the formation of a second PB.

However, here neither division of the first PB, as in $D$. coronatus, nor refusion with the oocyte nucleus, as in $A$. nanus, takes place. Our observation that the oocyte nucleus in Plectus sp. grows to a size equivalent to that of both pronuclei in C. elegans (see Fig. 2 B,D) suggests that also eggs of this species reach a diploid status prior to first cleavage. Whether this is accomplished via an additional DNA replication round during the approximately $100 \mathrm{~min}$ between egg-laying and first cleavage remains to be determined.

\section{Establishment of polarity, initiation of cleavage and orientation of the a-p axis}

In $C$. elegans the oocyte is fertilized at the pole that enters the spermatheca first and thus embryos cleaving in the uterus point with their posterior pole toward the vulva (Fig. 4A; see introduction).

Like $C$. elegans the three parthenogenetic representatives studied here express a polarity in the early embryo. With their first cleavage they generate a larger somatic cell $A B$ and a smaller germline cell $P_{1}$. All three species normally lay their eggs as softshelled 1-cell stages, which are severely squeezed while passing through the vulva (Fig. 5A). Only afterward do eggshells become solid. This is in contrast to $C$. elegans where embryos perform several cleavages inside the uterus before they are laid. Thus, in the former cases it cannot be readily determined whether the establishment of the embryonic anteriorposterior axis takes place within the gonadal tube of the mother or whether the laying process going along with massive egg deformation is crucial for axis specification. To investigate this question, we blocked egg-laying with a drug that allowed continuation of egg production such that several eggs accumulated in the uterus.

Under these conditions, in $A$. nanus and $D$. coronatus eggs performed the typical asymmetric cleavages inside the gonadal tube. Therefore, in these species the fixation of the a-p polarity can occur inside the mother prior to egg-laying. nuclei.
In contrast, eggs of Plectus sp. arrest before first cleavage under these conditions. This is probably not due to the applied drug as we found a similar situation in old mothers which had lost their ability to lay eggs. DNA staining revealed that all embryos were arrested in meiosis II. When such experimentally accumulated eggs were cut out of Plectus females, the most mature of them located close to the vulva completed meiosis and passed through normal embryogenesis, demonstrating that the developmental block is reversible.

Not only in experimental embryos but also in untreated Plectus females cultured on agar plates a variable fraction of laid eggs (sometimes the majority) arrested at identical positions in meiosis II (Fig. 3F). Although normal looking with respect to size and shape they never started cleavage.

In the two species where we could readily test it, we investigated whether the orientation of the a-p axis is the same as in $C$. elegans (see above). In $A$. nanus we found that - as in C. elegans-98\% showed a preferred orientation in the gonadal tube $(n=98)$, however, with opposite orientation to $C$. elegans (Fig. 4B). Only $2 \%$ pointed with their posterior pole toward the
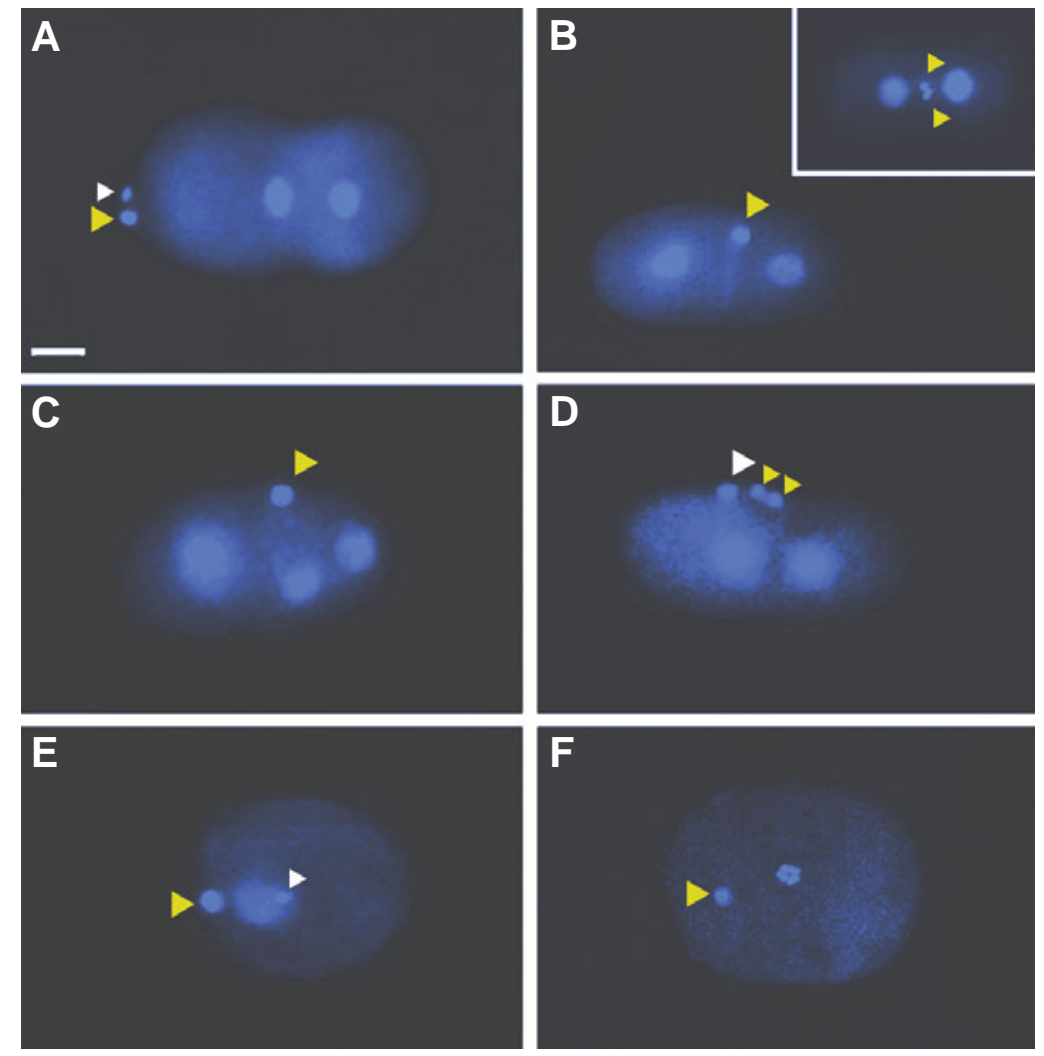

Fig. 3. Meiosis and formation of polar bodies (PBs). (A) C. elegans, uncleaved egg prior to fusion of pronuclei; two PBs have been extruded at the anterior pole as the result of two consecutive meiotic divisions. (B) D. coronatus, 2-cell stage, one PB present; insert: 2-cell embryo after division of the single PB. (C) A. nanus, 3-cell stage, one PB present. (D) Acrobeloides sp. (PS1146), hermaphrodite, 2-cell stage with 3 PBs. (E) Plectus sp. (ES 601) 1-cell stage with two PBs produced in two meiotic divisions. (F) Plectus sp. (ES 601), 1-cell stage, arrest in meiosis /l after one PB lout of focus) has been formed. Arrowheads (yellow), first $P B$ or its descendants; arrowheads (white), second PB; bar, $10 \mu \mathrm{m}$; Epifluorescence visualizing DAPI-stained 

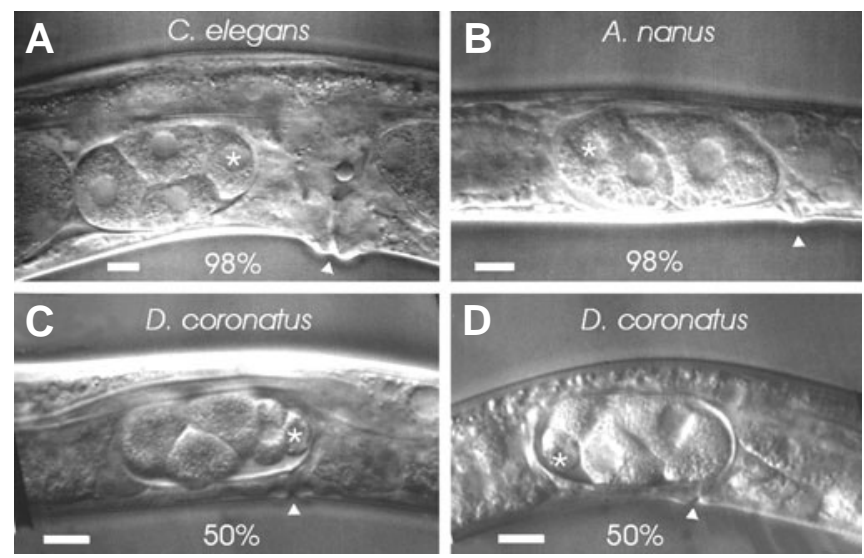

Fig. 4. Establishment of embryonic polarity. (A) C. elegans, posterior pole toward the vulva. (B) A. nanus, anterior pole toward the vulva. (C) D. coronatus, posterior pole toward the vulva. (D) D. coronatus, anterior pole toward the vulva. Arrowheads, position of the vulva; asterisks, germline cells $P_{2}(A, B)$ or $P_{3}(C, D)$, respectively, defining the posterior pole; $\%$, proportion of embryos behaving as shown; bars, $10 \mu \mathrm{m}$; Nomarski optics.

vulva. Thus, it appears that in $A$. nanussome external cue other than sperm induces the direction of egg polarity.

Eggs of $D$. coronatus behave differently. We found that half of them (54/107) point with their anterior pole toward the vulva but the other half with their posterior pole (Fig. 4 C,D). Here, the fixation of anterior-posterior polarity seems to be independent of an external signal and determined randomly by chance.

In Plectus sp. where cleavage could not be induced to take place within the mother (see above) we have not been able to relate polarity of embryos to a specific orientation of eggs in the gonadal tube. Reasons are deformations during egg laying, vigorous cytoplasmic streaming before first cleavage and variable orientation of the embryonic a-p axis relative to the only slightly oval eggshell (Lahl et al., 2003).

Looking for visible hints for such an event imprinting egg polarity in parthenogenetic nematodes, we traced the development of individual oocytes within the mother. We observed that in $A$. nanus (with fixed orientation) but not in $D$. coronatus (with variable orientation) eggs are squeezed through a narrow section in the $U$-shaped transition zone between the distal and the proximal section of the gonadal tube (Fig. 5B; $n$ $=6$ ), resulting in a temporary contact between nucleus and the cell cortex.

\section{Discussion}

All of the studied nematode embryos express an overt early polarity which can be easily visualized by the asymmetric first cleavage generating a larger anterior and a smaller posterior cell. However, the way of how this polarity is established appears to differ not only between $C$. elegans and the parthenogenetic species but also among the latter.

In $C$. elegans a small fraction of eggs shows an inverted polarity (Albertson, 1984; our data: 2/102), indicating a sperm entrance at the distal rather than the proximal pole of the oocyte, a situation that can also be induced experimentally (Goldstein and Hird, 1996). Analyzing uncleaved eggs that had been experimentally prevented from being laid we found that despite the absence of sperm in $A$. nanus, $98 \%$ of eggs express a defined anterior-posterior polarity (although with opposite orientation to C. elegans; see Fig. 4), suggesting that also in this parthenogenetic species a distinct, binary mechanism is acting with a close-to-perfect fidelity. Similar observations have been made by Goldstein et al. (1998) in a hermaphroditic Acrobeloides species where the position of the sperm bears no consistent relationship to embryonic polarity. They suggested that in cephalobid nematodes, independent of the mode of reproduction, a mechanism distinct from that in $C$. elegans has evolved to establish embryonic polarity.

Different is the case of $D$. coronatus, where fixation of axis polarity appears to be random. Consistent with the variations between $A$. nanus and $D$. coronatus concerning polarity formation, in the former but not in the latter we observed a massive temporary distortion of oocytes in the gonadal tube. Evidence has been presented that in certain insects distortion of unfertilized eggs in the ovipositor is sufficient to activate them and establish a primary polarity (Went and Krause, 1973).

As eggs of Plectus sp. arrest as 1-cell stages if prevented from being laid we have not been able to study in detail establishment of polarity in this species. From looking at the pattern of intracellular rearrangements Goldstein et al. (1998) suggested for another parthenogenetic Plectus species that with respect to axis specification the oocyte nucleus may take over the function of the sperm nucleus in $C$. elegans.

Although suggestive, it remains to be demonstrated that parthenogenetic species make use of the same general mechanism for axis specification as found in $C$. elegans, i.e. through interaction between the nucleus-associated centrosome, which in these cases would have to come from the oocyte rather than the sperm, and the cortex (Wallenfang and Seydoux, 2000; Cowan and Hyman, 2004). If this was true, one would have to postulate a polarizing event acting before that causing the oocyte nucleus and/or centrosome to move to a peripheral position. In conjunction with meiosis we observed such a nuclear movement. However, in contrast to $C$. elegans, in our parthenogenetic species the region of polar body formation does usually not coincide with the future posterior or anterior region of the egg (see Fig. 3).

A switch in environmental conditions (e.g. inside vs. outside of the mother or host) is apparently mandatory for the initiation of cleavage in a variety of free-living (e.g. Enoplus; Malakhov, 1994) and parasitic (e.g. Ascaris; Boveri, 1899) nematodes.
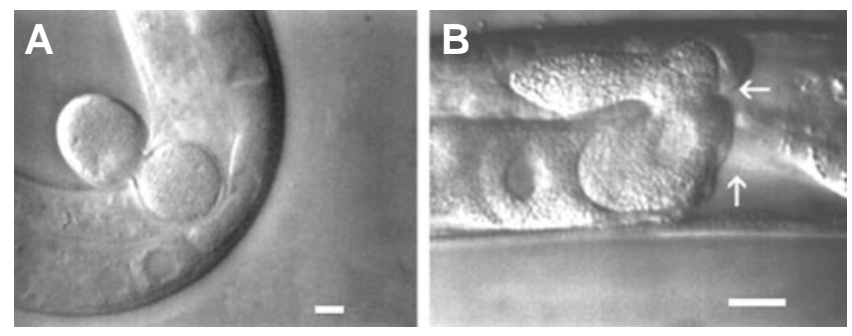

Fig. 5. Deformation of $\boldsymbol{A}$. nanus eggs prior to first cleavage. (A) Uncleaved egg squeezed through the vulva. (B) Egg cell squeezed during passage through the gonadal tube; arrows, direction of egg cell movement from distal to proximal (toward vulva); bars, $10 \mu \mathrm{m}$; Nomarski optics. 
While it is conceivable that such a strategy is favorable for parasitic species where infection of new hosts is required, the advantage for free-living species is less obvious and may indicate a strategy where the mother senses suitable environmental conditions for egg deposition.

We found that in Plectus sp. egg cells inside the mother reversibly arrest before first cleavage. This indicates that at least in this parthenogenetic species a meiotic block exists like in $C$. elegans, where it is released through a sperm-mediated signal (Miller etal., 2001; Hajnal and Berset, 2002). Surprisingly, also a major proportion of laid eggs remains permanently arrested in meiosis. It remains to be analyzed which factors are responsible here for releasing the meiotic block and why such a crucial event takes place - at least under our laboratory conditions - only in part of the eggs.

In conclusion, our findings that even closely related nematode species reproduce differently and that parthenogenetic representatives express distinct variations in their effort to compensate for functions which in other species are taken over by the sperm support the view that the basic developmental program contains a considerable degree of plasticity and that parthenogenesis arose multiple times independently within the phylum Nematoda.

\section{Materials and Methods}

\section{Nematode strains}

Studies were carried out with Caenorhabditis elegans strain N2 (hermaphroditic reproduction), Acrobeloides nanus (strain designation ES501; formerly named Cephalobus sp.; Skiba and Schierenberg, 1992); Plectus sp. (ES 601; Lahl et al., 2003); Diploscapter coronatus (PDL 0010; kindly provided by Paul de Ley, Riverside, CA). In addition, two other species, Acrobeloides sp. (PS 1146; hermaphroditic reproduction; kindly provided by Marie-Anne Felix, Paris, France and Acrobeloides sp. (SB 374; dioecious reproduction; kindly provided by Walter Sudhaus, Berlin, Germany) were tested for comparison. For phylogenetic positions of the studied nematodes, see Fig. 1. Phylogenetic nomenclature is according to De Ley and Blaxter (2002).

\section{Nematode culture}

Nematode strains were cultured on agar plates with the uracilrequiring strain of $E$. coli OP50 as a food source, essentially as described by Brenner (1974) except that, to keep contamination with other bacteria to a minimum, we used low-salt plates (Lahl et al., 2003). This results in a thinner than normal bacterial lawn. Plectus species require low-salt conditions.

\section{Microscopical analysis}

Living adults were mounted on microscope slides carrying thin 5\% agar pads as described in Irle and Schierenberg, 2002. Preparations of living embryos on $3 \%$ agar-coated slides were performed according to Lahl et al. (2003). For fluorescent-dye staining of DNA adults and embryos were placed on polylysine-coated slides, fixed with methanol/ acetone and stained with DAPI $(0.1 \mu \mathrm{g} / \mathrm{ml})$. In selected cases, continuous video recordings under Nomarski optics were made of oocytes inside the mother and subsequent cleavage at least up to the 4-cell stage.

\section{Drug treatment}

To prevent egg-laying, mothers were placed in PBS containing $0.03-0.05 \% \mathrm{NaN}_{3}$. This inhibition (originally designed to narcotize worms; Sulston and Hodgkin, 1988; modified) is reversible after trans- fer of animals back to agar plates. Our initial experiments revealed some species-specific variations with respect to drug sensitivity.

\section{Acknowledgments}

We thank Walter Sudhaus, Paul De Ley and Marie-Anne Felix for nematode strains, Randy Cassada for helpful comments, Paul De Ley for discussion, Elisabeth Trojtza for skillful technical assistance and the Deutsche Forschungsgemeinschaft for support.

\section{References}

ALBERTSON, D. G. (1984). Formation of the first cleavage spindle in nematode embryos. Dev. Biol. 101: 61-72.

ALBERTSON, D.G. and THOMSON, J.N. (1993). Segregation of holocentric chromosomes at meiosis in the nematode Caenorhabditis elegans. Chromosome Res. 1:15-26.

BOVERI, T. (1899). Die Entwicklung von Ascaris megalocephala mit besonderer Rücksicht auf die Kernverhältnisse. Festschrift C. von Kupffer, Jena, Gustav Fischer Verlag, pp. 383-430.

BRENNER, S. (1974). The genetics of Caenorhabditis elegans. Genetics 77: 7194.

COLE, C.J. and TOWNSEND, C.R. (1990). Parthenogenetic lizards as vertebrate systems. J. Exp. Zool. Suppl. 4: 174-176.

COWAN, C.R. and HYMAN, A.A. (2004). Centrosomes direct cell polarity independently of microtubule assembly in C. elegans. Nature 431: 92-96

DE LEY, P. and BLAXTER, M. (2002). Systematic position and phylogeny. In The biology of nematodes. (Ed. Lee, D.L.). Taylor and Francis, London, pp. 1-30.

GOLDSTEIN, B. and HIRD, S.N. (1996) Specification of the anteroposterior axis in Caenorhabditis elegans. Development 122: 1467-1474.

GOLDSTEIN, B., FRISSE, L.M. and THOMAS, W.K. (1998). Embryonic axis specification in nematodes: evolution of the first step in development. Curr. Biol. 8: 157-160.

HAJNAL, A. and BERSET, T. (2002). The C.elegans MAPK phosphatase LIP-1 is required for the $\mathrm{G}(2) / \mathrm{M}$ meiotic arrest of developing oocytes. EMBO J.21: 4317-4326.

HAMILTON, W.D., AXELROD, R. and TANESE, R. (1990). Sexual reproduction as an adaptation to resist parasites. Proc. Nat/. Acad. Sci. USA 87: 35663573.

IRLE, T. and SCHIERENBERG, E. (2002) Developmental potential of fused $C$. elegans oocytes: generation of giant and twin embryos. Dev. Genes Evol. 212: $257-266$

KONO, T., OBATA, Y., WU, Q., Niwa, K., ONO, Y., YAMAMOTO, Y., PARK, E.S., SEO, J.S. and OGAWA, H. (2004). Birth of parthenogenetic mice that can develop to adulthood. Nature 428: 860-864.

LAHL, V., HALAMA, C. and SCHIERENBERG, E. (2003). Comparative and experimental embryogenesis of Plectidae (Nematoda). Dev. Genes Evol. 213: $18-27$.

MALAKHOV, V.V.(1994). Nematodes, Structure, Development, Classification and Phylogeny. Washington, D.C., USA, Smithsonian Institution Press.

MAYNARD-SMITH, J. (1978). The Evolution of Sex. Cambridge, University Press.

MILLER, M.A., NGUYEN, V.Q., LEE, M.-H., KOSINSKI, M., SCHEDL, T., CAPRIOLI, R.M. and GREENSTEIN, D. (2001). A sperm cytoskeletal protein that signals oocyte meiotic maturation and ovulation. Science 291: 21442147.

MITTWOCH, U. (1978) Parthenogenesis. J. Med. Genet.15:165-181.

NIGON, V., GUERRIER, P. and MONIN, H. (1960). L'architecture polaire de l'oeuf et les mouvements des constituents cellulaire au cours des premières étappes du développement chez quelques nématodes. Bull. Biol. Fr. Belg. 94: 131-202.

SKIBA, F. and SCHIERENBERG, E. (1992). Cell lineages, developmental timing 
398 V. Lahl et al.

and spatial pattern formation in embryos of free-living soil nematodes. Dev. Biol. 151: 597-610.

SULSTON, J. and HODGKIN, J. (1988). Methods. In: Wood, W.B. (ed.) The nematode Caenorhabditis elegans. Cold Spring Harbor Laboratory, Cold Spring Harbor, pp. 587-606.

VINEY, M. (1999). Exploiting the life cycle of Strongyloides ratti. Parasitol. Today 15: 231-235.

WALLENFANG, M.R. and SEYDOUX, G. (2000). Polarization of the anteriorposterior axis of $C$. elegans is a microtubule-directed process. Nature 408: 89-92.
WENT, D.F. and KRAUSE, G. (1973). Normal development of mechanically activated, unlaid eggs of an endoparasitic hymenopteran. Nature 244: 454455.

Received: May 2005

Reviewed by Referees: September 2005

Modified by Authors and Accepted for Publication: November 2005 